\title{
Non-recurrent meromorphic functions
}

\author{
by \\ Jacek Graczyk (Orsay), Janina Kotus (Warszawa) \\ and Grzegorz Świątek (University Park, PA)
}

To the memory of I. N. Baker

\begin{abstract}
We consider a transcendental meromorphic function $f$ belonging to the class $\mathcal{B}$ (with bounded set of singular values). We show that if the Julia set $J(f)$ is the whole complex plane $\mathbb{C}$, and the closure of the postcritical set $P(f)$ is contained in $B(0, R) \cup\{\infty\}$ and is disjoint from the set Crit $(f)$ of critical points, then every compact and forward invariant set is hyperbolic, provided that it is disjoint from Crit $(f)$. It is further shown, under general additional hypotheses, that $f$ admits no measurable invariant line-field.
\end{abstract}

1. Introduction. The orbits of points under iteration by a meromorphic function fall into three categories: they may be infinite, they may become periodic and hence consist of a finite number of distinct points, or they may terminate at a pole of the function. Points in the last category are called prepoles. For transcendental meromorphic functions with more than one pole, it follows from Picard's theorem that there are infinitely many prepoles.

The Fatou set $F(f)$ of a meromorphic function $f: \mathbb{C} \rightarrow \overline{\mathbb{C}}$ is defined in exactly the same manner as for rational functions: it is the set of points $z \in \mathbb{C}$ such that all the iterates are defined and form a normal family in a neighborhood of $z$. The Julia set $J(f)$ is the complement of $F(f)$ in $\overline{\mathbb{C}}$. Thus, $F(f)$ is open, $J(f)$ is closed, $F(f)$ is completely invariant, while $f^{-1}(J(f))=J(f) \backslash\{\infty\}$ and $f(J(f) \backslash\{\infty\}) \subset J(f)$. For a description of the dynamics of meromorphic functions see e.g. [3]. We note, however, that a meromorphic function which is not entire either has exactly one pole $w$ and $w \notin f(\mathbb{C})$ and $f$ is a self-map of the punctured plane, i.e. $f: \mathbb{C} \backslash\{w\} \rightarrow \mathbb{C} \backslash\{w\}$, or $f: \mathbb{C} \rightarrow \overline{\mathbb{C}}$ has at least one pole which is not an

2000 Mathematics Subject Classification: Primary 37F50; Secondary 30D05.

Research of J. Kotus partially supported by a KBN grant No. 2 PO3A 03425.

Research of G. Świątek partially supported by an NSF grant DMS-0245358. 
omitted value and then (see [1])

$$
J(f)=\overline{\bigcup_{n \geq 0} f^{-n}(\infty) .}
$$

Recall that $a \in \overline{\mathbb{C}}$ is an asymptotic value of a meromorphic function $f$ provided that there is a curve $\Gamma \subset \mathbb{C}$ tending to $\infty$ such that $f(z) \rightarrow a$ as $z \rightarrow \infty, z \in \Gamma$. Such a $\Gamma$ is called an asymptotic curve. The notions of a critical point and critical value need no restatement and a singular value will refer to either an asymptotic or a critical value.

A transcendental meromorphic function may omit some values (for example $f(z)=e^{z}$ omits $0, \infty$, and $\tan (z)$ omits $\left.\pm i\right)$. By Picard's theorem the number of omitted values cannot exceed 2 and by Iversen's theorem each of them is an asymptotic value (see [6]).

Notation. Throughout the paper we denote by Crit $(f)$ the set of critical points which are not multiple poles of $f$. By $\operatorname{Sing}(f)$ we denote the set of finite singular values of $f$, i.e. $c \in \operatorname{Sing}(f)$ if $c \in \mathbb{C}$ and $c$ is a critical or asymptotic value of $f$. We also consider the post-singular set defined as

$$
P(f):=\bigcup_{n=0}^{\infty} f^{n}(\operatorname{Sing}(f)) .
$$

Here and elsewhere $f^{n}(A)$ is the set of points in $\overline{\mathbb{C}}$ which have a preimage in $A$ under $f^{n}$, for example $f(\infty)=\emptyset$.

Definition 1. A set $K \subset \mathbb{C}$ is called $n$-regular if for every $c \in \operatorname{Sing}(f)$ and every $k \in \mathbb{N}$ such that $k \leq n$ and $f^{k}(c) \in K$, there exists an inverse branch $g$ of $f^{k}$ defined on $K$ such that $g\left(f^{k}(c)\right)=c$.

Definition 2. A set $K \subset \mathbb{C}$ is called regular if it is $n$-regular for every $n \in \mathbb{N}$.

A set $K \subset \mathbb{C}$ is called hyperbolic if there are $N \in \mathbb{N}$ and $k>1$ such that $\left|\left(f^{N}\right)^{\prime}(x)\right|>k$ for every $x \in K$, provided that $f^{N}$ is defined at $x$.

In this paper diam $K$ denotes the Euclidean diameter of the set $K$, and $\bmod (L)$ is the modulus of an annulus $L$.

Let $\mathcal{S}:=\{f: \mathbb{C} \rightarrow \overline{\mathbb{C}}: \operatorname{Sing}(f)$ is finite $\}$ and $\mathcal{B}:=\{f: \mathbb{C} \rightarrow \overline{\mathbb{C}}:$ $\operatorname{Sing}(f)$ is bounded $\}$. The class $\mathcal{B}$ has been studied by many people. However, the dynamics of maps in the class $\mathcal{B}$ is richer and consequently less understood than for $\mathcal{S}$. For example in $\mathcal{S}$ the classification of periodic components is the same as for rational maps, which is not the case for $\mathcal{B}$ (see [3], [2] and $[8])$.

$B(x, \varepsilon)$ and $C(x, \varepsilon)$ will refer to the open Euclidean disk and circle of center and radius given, respectively. 
Misiurewicz condition. The following will be referred to as the Misiurewicz condition: $P(f) \subset B(0, R) \cup\{\infty\}$ for some $R>0$ and $\overline{P(f)} \cap \operatorname{Crit}(f)$ $=\emptyset$.

The Misiurewicz condition comes from interval dynamics, where it simply says that $P(f)$ is disjoint from $\operatorname{Crit}(f)$. It was later used in the study of dynamics of rational maps and is widely viewed as the first generalization going beyond the realm of post-critically finite maps. In the case of meromorphic dynamics which we are dealing with here, $\infty$ can be viewed as a critical point of sorts with asymptotic values akin to critical values arising from it. Then our condition is tantamount to the classical Misiurewicz condition, with one exception that falling into $\infty$ is allowed.

We shall prove the following.

TheOREM 1. Let $f: \mathbb{C} \rightarrow \overline{\mathbb{C}}$ be a transcendental meromorphic function in the class $\mathcal{B}$ such that $J(f)=\overline{\mathbb{C}}$. If additionally $f$ satisfies the Misiurewicz condition then every compact and forward invariant set $K$, disjoint from the set $\operatorname{Crit}(f)$ of critical points, is hyperbolic.

For rational mappings Theorem 1 is known to follow from the work of Mañé, although it has not been stated in the literature. In the meromorphic case, a somewhat different method has to be used.

The proof of Theorem 1 comes in the next section. In Section 3 we will give another theorem, under some additional hypotheses, about nonexistence of invariant line-fields.

2. Proof of Theorem 1. Suppose that the hypotheses of Theorem 1 hold.

Lemma 1. For every $R>0$ and every $x \in \mathbb{C}$ there is $\varepsilon>0$ such that every connected component $D_{i}$ of $f^{-1}(B(x, \varepsilon))$ with $\left.f^{-1}(B(x, \varepsilon)) \cap \overline{B(0, R}\right)$ $\neq \emptyset$ is a topological disk containing exactly one preimage of $x$ and $f$ : $D_{i} \rightarrow B(x, \varepsilon)$ is a proper map.

Proof. We fix $R>0$ and $x \in \mathbb{C}$. If $R_{1}>R$, for $\varepsilon$ small enough all connected components of $f^{-1}(B(x, \varepsilon))$ which intersect $B(0, R)$ are contained in $B\left(0, R_{1}\right)$, otherwise we would end up with a preimage of $x$ on every circle with radius between $R$ and $R_{1}$. By continuity of $f$, the boundary of each such component $D_{i}$ is mapped into $C(x, \varepsilon)$. Hence, $f_{\mid D_{i}}$ is proper onto $B(x, \varepsilon)$, in particular it contains a preimage $x_{i}$ of $x$. So, there are only finitely many components $D_{i}$ and, possibly for smaller $\varepsilon$, each contains exactly one preimage of $x$. From the local form of an analytic function, for $\varepsilon$ small enough each $D_{i}$ is a topological disk.

Lemma 2. For every $\varepsilon>0, M>0$, and $R>0$ there is $\delta>0$ such that for every $n \geq 1$ if $U, K \subset \mathbb{C}, K \subset U, U$ is a topological disk while $K$ is 
compact and simply connected, $\bmod (U \backslash K) \geq M$, $\operatorname{diam} K<\delta$, and $g$ is an analytic branch of $f^{-n}$ defined on $U$ such that $\operatorname{dist}(g(K), 0)<R$, then $\operatorname{diam} g(K)<\varepsilon$.

Proof. Let $M, R, \varepsilon>0$ be given and suppose that the assertion does not hold. Then choose a sequence of triples $\left(U_{p}, K_{p}, g_{p}\right)$ with diam $K_{p} \rightarrow 0$ such that $g_{p}: U_{p} \rightarrow \mathbb{C}$ is an inverse branch of $f^{n_{p}}$ and $\operatorname{diam} g_{p}\left(K_{p}\right) \geq \varepsilon>0$. Also, make sure that $\operatorname{dist}\left(0, g_{p}\left(K_{p}\right)\right)<R$ and $\bmod \left(U_{p} \backslash K_{p}\right) \geq 2 M$ for every $p$. By decreasing each $U_{p}$ and using the Koebe distortion theorem, we may assume without loss of generality that the distortion of $g_{p}$ on $U_{p}$ is bounded uniformly in $p, g_{p}\left(U_{p}\right)$ are quasi-disks of uniform distortion and $\bmod \left(U_{p} \backslash K_{p}\right) \geq M$. Since the diameters of $K_{p}$ go to 0 , but the diameters of $g_{p}\left(K_{p}\right)$ stay bounded away from 0 , inf $\left\{\left|g_{p}^{\prime}(z)\right|: z \in U_{p}\right\} \rightarrow \infty$ as $p \rightarrow \infty$.

There is $\eta>0$, depending on $\varepsilon$ and $M$, such that for every $p$ the set $g_{p}\left(U_{p}\right)$ contains a ball of radius $\eta$ which intersects $B(0, R)$. By taking an accumulation point of the centers of such balls for various $p$, we find a ball of radius $\eta / 2$ which is contained in $g_{p}\left(U_{p}\right)$ for infinitely many $p$, without loss of generality for all $p$. On this ball, we get a sequence of iterates $f^{n_{p}}$ with derivatives tending uniformly toward 0 . This means that $n_{p}$ tends to $\infty$ with $p$. Then, however, we get a contradiction with the density of repelling periodic orbits in the Julia set, which in our case is the whole plane.

The proof of Lemma 2 is one of few places where the hypothesis that the Julia set is the whole sphere gets used. One might expect by analogy with the rational case that an assumption of that type is superfluous. Lemma 2, however, obviously fails even in the rational case if the Fatou set contains attracting or parabolic basins, so we have to leave that issue open for now.

LEMMA 3. For every $R, \eta>0$ there is $\delta>0$ such that for every $x, y \in$ $B(0, R)$ if $x=f(y)$ and $\operatorname{dist}(y, \operatorname{Crit}(f)) \geq \eta$, then there is an inverse branch $g$ of $f$ defined on $B(x, \delta)$ such that $g(x)=y$.

Proof. Define

$$
K:=\overline{B(0, R)} \backslash f(\{z: \operatorname{dist}(z, \operatorname{Crit}(f))<\eta\}) .
$$

Since $f$ is an open mapping, $K$ is compact. Using Lemma 1, for every $y \in K$ we can find $\varepsilon(y)>0$ such that the connected components of $f^{-1}(B(y, \varepsilon(y)))$ which intersect $B(0, R)$ are topological disks of diameter less than $\eta / 2$. The balls $B(y, \varepsilon(y)), y \in K$, form an open covering of $K$; let $\delta>0$ be its Lebesgue number. That is, every ball of radius $\delta$ and center in $K$ is contained in one element of the covering. Then, if $x=f(y)$ satisfies the hypothesis of Lemma 3, there is $z \in K$ such that $B(x, \delta) \subset B(z, \varepsilon(z))$. In particular, $y$ belongs to a connected component of $f^{-1}(B(z, \varepsilon(z)))$ which is disjoint from Crit $(f)$. Then the inverse branch is defined on $B(z, \varepsilon(z))$. 
Lemma 4. Let $U$ be a topological disk in the plane, and $K \subset U$ be compact and simply connected such that $\bmod (U \backslash K) \geq M$. Suppose that $K_{i}$, $i=0, \ldots, m$, are simply connected sets which satisfy $K_{0}=K, f\left(K_{i}\right)=K_{i-1}$ for $i=1, \ldots, m$. Analogously, define $U_{i}$ to be the connected component of $f^{-i}(U)$ which contains $K_{i}$. Assume also $K_{m} \cap P(f) \neq \emptyset$ and that there is an inverse branch $\gamma: U \rightarrow U_{m-1}$ of $f^{m-1}$ for which $\gamma(K)=K_{m-1}$.

For every $M>0$ there is $\varepsilon>0$ such that if $\operatorname{diam} U<\varepsilon$ and $W$ is a topological disk with $K \subset W \subset \bar{W} \subset U$ and $\bmod (U \backslash \bar{W}) \geq M / 2$, then there is an inverse branch $g: W \rightarrow U_{m}$ of $f^{m}$ such that $g(K)=K_{m}$.

Proof. By Lemma 2 for every $\delta>0$ we can find $\varepsilon>0$ such that $\operatorname{diam} \gamma(W)<\delta$. Now, the connected component of $\gamma(W)$ which contains $K_{m-1}$ also intersects $P(f)$, hence we can use Lemma 3 and the Misiurewicz condition to choose $\delta$ in such a way that $f$ has an inverse branch defined on $\gamma(W)$ which maps $K_{m-1}$ to $K_{m}$.

Lemma 5. Let $U$ be a topological disk in the complex plane, and $K \subset U$ be compact and simply connected. Let $\left(P_{i}\right)_{i=1}^{N}$ be a finite collection of open connected sets with union $U \backslash K$. Consider another topological disk $K \subset$ $W \subset U$ and assume that there is an inverse branch $g: W \rightarrow \mathbb{C}$ of $f$. Suppose also that $P_{i} \cap W$ is connected for all $i$. Let $S_{i}$ be the connected component of $f^{-1}\left(P_{i}\right)$ which contains $g\left(P_{i}\right)$. Then the following holds:

- If $f: S_{i} \rightarrow P_{i}$ is univalent for each $i=1, \ldots, N$, then $g$ has an analytic continuation defined on $U$.

- If $f: S_{i} \rightarrow P_{i}$ is not univalent and $P_{i}$ is n-regular for some $n$ (see Definition 1$)$, then $S_{i}$ does not contain any point of the form $f^{k}(c)$, where $c \in \operatorname{Sing}(f)$ and $k<n$.

Proof. We will rely on the following criterion of univalence. If $C, D \subset \mathbb{C}$ are open and $D$ is simply connected, then $f: C \rightarrow D$ is univalent if and only if $f$ is proper and its derivative does not vanish on $C$.

To prove the first claim consider $C=g(W) \cup \bigcup_{i=1}^{N} S_{i}$. From the hypothesis, the derivative does not vanish on $C$. To check that the map is proper, we can represent any compact set relatively compact in $U$ as the union of sets, each relatively compact in some $P_{i}$ or in $W$. Then we use the fact that the preimage of any of these sets is compact in $C$.

To see the second claim, proceed by contradiction and assume that $f^{k}(c) \in S_{i}$ for some $c \in \operatorname{Sing}(f)$ and $k<n$. The regularity of $P_{i}$ means that there is an inverse branch $\gamma$ of $f^{k+1}$ whose domain is $P_{i}$ and whose image contains $c$. Then $f \circ\left(f^{k} \circ \gamma\right)$ is the identity. Thus, $f$ is injective and onto as a map from $f^{k}\left(\gamma\left(P_{i}\right)\right) \ni f^{k}(c)$ onto $P_{i}$. Since the first of these sets intersects $S_{i}$, we get $f^{k}\left(\gamma\left(P_{i}\right)\right)=S_{i}$ and $f$ is univalent, contrary to the hypothesis. 
Proposition 1. Let $0<r<R$ and $z_{0} \in \mathbb{C}$. Suppose that an inverse branch $g$ of $f^{n}$, for some $n \geq 1$, is defined on a neighborhood of $\overline{B\left(z_{0}, r\right)}$, and that $g\left(B\left(z_{0}, r\right)\right) \cap P(f) \neq \emptyset$. Suppose also that the annulus $B\left(z_{0}, R\right) \backslash \overline{B\left(z_{0}, r\right)}$ is the union of finitely many $n$-regular sets $P_{i}, i=1, \ldots, N$, each of which is a "rectangle" bounded by arcs of $C\left(z_{0}, r\right), C\left(z_{0}, R\right)$, and two radii of $B\left(z_{0}, R\right)$.

For every $\eta>1$ and $N$ there are $\delta>0$ and $\varepsilon>0$, independent of $n$, such that whenever $R / r \geq 1+\eta$ and $R<\varepsilon$, then $g$ has an analytic continuation to $B\left(z_{0},(1+\delta) r\right)$.

Proof. In the setting of Proposition 1, we will refer to a set $P_{i}$ as postsingular provided that for some $k \leq n$ there is no inverse branch of $f^{k}$ which sends $P_{i}$ into the connected component of $f^{-k}\left(B\left(z_{0}, R\right)\right)$ which contains $f^{n-k}\left(g\left(B\left(z_{0}, r\right)\right)\right)$. Let $M$ denote the number of post-singular sets among $P_{i}$, $i=1, \ldots, N$.

We apply induction on $M$. For $M=0$, one immediately constructs the extension of $g$.

Suppose now that $M>0$ and let $m$ be the maximal integer in $\{1, \ldots, n\}$ such that $f^{m-1}$ has an inverse branch $F$ defined on $B\left(z_{0}, R\right)$ which continues $f^{n-m+1} \circ g$. Then we are in the situation of Lemma 4 . It implies that when $\varepsilon$ is suitably small, then the appropriate inverse branch of $f^{m}$ can still be defined on $B\left(z_{0}, \sqrt{r R}\right)$.

Invoking Lemma 5 , we see that either $f$ is not proper or it has a critical point on a set $S_{i_{0}}$. Here $S_{i_{0}}$ is the connected component of $f^{-1}\left(F\left(P_{i_{0}}\right)\right)$ which contains the image of $P_{i_{0}} \cap B\left(z_{0}, \sqrt{r R}\right)$ under the inverse branch of $f^{m}$ constructed in the previous paragraph. Observe here that the sets $F\left(P_{i}\right)$ are $n-m+1$-regular. But then the second claim of Lemma 5 says that $S_{i_{0}}$ does not contain any point $f^{k}(c)$ with $c \in \operatorname{Sing}(f)$ and $k<$ $n-m+1$.

Now replace $B\left(z_{0}, R\right)$ with $B\left(z_{0}, \sqrt{R r}\right)$ in the statement of Proposition 1 and consider the "trimmed" sets $P_{i}^{\prime}:=P_{i} \cap B\left(z_{0}, \sqrt{R r}\right)$. Let $M^{\prime}$ denote the number of post-singular sets among the $P_{i}^{\prime}$. Clearly, if $P_{i}^{\prime}$ is post-singular in this new setting, it was also post-singular before, thus $M^{\prime} \leq M$ with equality possible only if all $P_{i}$ which were post-singular also give rise to post-singular $P_{i}^{\prime}$.

However, we will now show that $P_{i_{0}}^{\prime}$ is not post-singular. Checking the condition in the definition of the post-singular set, we see that it cannot be satisfied with any $k \leq m$, since for such $k$ an inverse branch is defined globally on $B\left(z_{0}, \sqrt{r R}\right)$. But such an inverse branch has its image inside $S_{i_{0}}$, and by the second claim of Lemma $5, S_{i_{0}}$ does not contain any point $f^{j}(c)$ with $c \in \operatorname{Sing}(f)$ and $j<n-m$. Hence, all inverse branches of $f^{n-m}$ are well defined on $S_{i_{0}}$, which means that $P_{i_{0}}^{\prime}$ is not post-singular, as claimed. 
Thus, $M^{\prime}<M$, and Proposition 1 follows by induction.

Proposition 2. There is $\varepsilon>0$ such that for each $z_{0} \in \mathbb{C}$, the ball $B\left(z_{0}, \varepsilon\right)$ is regular.

Proof. Regularity means that $B\left(z_{0}, \varepsilon\right)$ is $n$-regular for every $n, z_{0}$. Of course, we have to be careful to choose $\varepsilon$ independently of $n$ or $z_{0}$.

0-regularity is a tautology. So assume that the statement holds for some $n \geq 0$. Pick a ball $B\left(z_{0}, \varepsilon\right)$. We can choose $R>\varepsilon$, fixed, in such a way that the annulus $B\left(z_{0}, R\right) \backslash \overline{B\left(z_{0}, \varepsilon\right)}$ can be covered by a fixed number $N$ of "rectangles" of the type described in Proposition 1, with each rectangle contained in a ball of radius $\varepsilon$. By elementary geometry $R=3 / 2 \varepsilon$ and $N=$ 100 will do. Suppose that $x \in B\left(z_{0}, \varepsilon\right)$ and $x=f^{n+1}(c)$ for some $c \in \operatorname{Sing}(f)$. To show that $B\left(z_{0}, \varepsilon\right)$ is $n+1$-regular, we only need to find an inverse branch of $f^{n+1}$ defined on $B\left(z_{0}, \varepsilon\right)$ which sends $x$ to $c$. On $B\left(z_{0}, \varepsilon(1+\delta)\right)$ with some fixed $\delta>0$ we can find an inverse branch of $f^{n}$ which maps $x$ to $f(c)$. This follows from Proposition 1 and requires $\varepsilon$ to be sufficiently small depending on $N$, but not $n$. Since these parameters can be fixed at $3 / 2$ and 100 , without loss of generality we can choose $\varepsilon$ as required by Proposition 1 and $\delta$ is fixed as well.

But now we can use Lemma 4 choosing $U=B\left(x_{0}, \varepsilon(1+\delta)\right)$ and $W=$ $B\left(x_{0}, \varepsilon \sqrt{1+\delta}\right)$ to deduce that again if $\varepsilon$ is sufficiently small depending on $\delta$, then $f^{n+1}$ has the appropriate inverse branch defined on $W \supset B\left(z_{0}, \varepsilon\right)$.

Proposition 2 now follows by induction.

\section{Hyperbolicity}

Lemma 6. Consider an inverse orbit $\left(x_{-n}\right)_{n=0}^{\infty}, x_{-n}=f\left(x_{-n-1}\right)$, and assume that the set $\left\{x_{-n}: n=0,1, \ldots\right\}$ is bounded in a disk $B(0, R)$ and its distance from $\operatorname{Crit}(f)$ is $\eta>0$. For every $R, \eta$ there is $\varepsilon>0$ such that for every $n$ there is an inverse branch of $f^{n}$ defined on $B\left(x_{0}, \varepsilon\right)$ which sends $x_{0}$ to $x_{-n}$.

Proof. First use Proposition 2 to choose $\varepsilon$ small enough so that $B\left(x_{0}, 2 \varepsilon\right) \backslash \overline{B\left(x_{0}, \varepsilon\right)}$ can be covered by "rectangles" in the sense of the hypothesis of Proposition 1 and those rectangles are all regular.

Next, invoke Lemma 3 to find $\delta>0$, depending on $R, \eta$, such that for every $n$ there is an inverse branch of $f$ defined on $B\left(x_{-n}, \delta\right)$ which sends $x_{-n}$ to $x_{-n-1}$.

Now proceed by induction. Suppose that an inverse branch $g_{n}$ of $f^{n}$ has been defined on $B\left(x_{0}, \varepsilon\right)$ and $g_{n}\left(x_{0}\right)=x_{-n}$. Use Proposition 1 with $r:=\varepsilon$ to conclude that $g_{n}$ can be continued to $B\left(z_{0}, r\right)$ with $r / \varepsilon>1$ and depending only the number of regular rectangles used to cover the annulus $B\left(x_{0}, 2 \varepsilon\right) \backslash \overline{B\left(x_{0}, \varepsilon\right)}$. Using Lemma 2 we see that if $\varepsilon$ is chosen small enough 
depending on $R$, hence independently of $n$, then diam $g_{n}\left(B\left(x_{0}, \varepsilon\right)\right)<\delta$. Now we can invert $f$ from $g_{n}\left(B\left(x_{0}, \varepsilon\right)\right)$ and so construct $g_{n+1}$.

Proposition 3. Let $K$ be a forward invariant compact set which does not intersect $\operatorname{Crit}(f)$. Then $K$ is hyperbolic.

Proof. We consider a backward orbit $\left(x_{n}\right)_{n=0}^{-\infty}$ in $K$. By Lemma 6 all inverse branches which follow this orbit are defined on the ball $B\left(x_{0}, \varepsilon\right)$, with $\varepsilon$ depending only on $K$ and not a particular orbit.

We will show that for every $\zeta>0$ there is $n \in \mathbb{N}$ such that for every inverse orbit $\left(x_{n}\right)_{n=0}^{-\infty}$ in $K$, if $g_{n}$ is a branch of $f^{-n}$ satisfying $g_{n}\left(x_{0}\right)=x_{-n}$ then $\operatorname{diam} g_{n}\left(B\left(x_{0}, \varepsilon / 2\right)\right)<\zeta$.

To the contrary, suppose there are $\zeta>0$ and a sequence of orbits $\left(x_{n}^{p}\right)$ and branches $g_{n_{k}}^{p}, n_{k} \rightarrow \infty$, of the inverse maps $f^{-n_{k}}$ such that

$$
\operatorname{diam} g_{n_{k}}^{p}\left(B\left(x_{0}^{p}, \varepsilon / 2\right)\right) \geq \zeta \text {. }
$$

By Koebe's Lemma, the distortion of $g_{n_{k}}^{p}$ is bounded on $B\left(x^{p}, \varepsilon / 2\right)$ uniformly in $p$, hence each of those sets contains $B\left(x_{n_{k}}^{p}, \zeta_{1}\right)$ for $\zeta_{1}>0$. Since the sets $g_{n_{k}}^{p}\left(B\left(x_{0}^{p}, \varepsilon / 2\right)\right)$ intersect a compact set $K$, there is a point $z$ such that $B\left(z, \zeta_{1} / 2\right)$ is contained in infinitely many of them. Then all the derivatives $\left(f^{n_{k}}\right)^{\prime}$ are uniformly bounded on some neighborhood of $z$, which contradicts the density of repelling periodic orbits in the Julia set.

If we choose $\zeta$ sufficiently small compared with $\varepsilon$, then the derivative of $g_{n}$ at $x_{0}$ is less than $1 / 2$ by Koebe's Quarter Theorem. Since $n, \zeta$ and $\varepsilon$ are uniform for all inverse orbits, we have $\left|\left(f^{n}\right)^{\prime}\right|>2$ uniformly on $K$.

Expansion to the large scale. As a final consequence, we deduce that under some extra hypotheses almost every point has a basis of neighborhoods each of which is mapped onto a ball of fixed radius by some iterate of $f$, and these balls do not escape to $\infty$.

Proposition 4. Suppose that $f$ is a meromorphic map whose Julia set is the whole plane, which satisfies the Misiurewicz condition and additionally $\infty \notin P(f)$. Also assume that the set of points whose orbits converge to $\infty$ has zero Lebesgue measure.

Then, for all $z \in \mathbb{C}$ except a set of zero Lebesgue measure, there exist $\varepsilon>0$ and a sequence $n_{k} \rightarrow \infty$ and a compact set $K \subset \mathbb{C}$ such that, for every $k, f^{n_{k}}(z) \subset K$ and there is a neighborhood of $z$ which is mapped univalently by $f^{n_{k}}$ onto $B\left(f^{n_{k}}(z), \varepsilon\right)$.

Proof. Before proving the proposition, observe that the derivatives $\left(f^{n_{k}}\right)^{\prime}(z)$ must diverge to $\infty$, because the repelling periodic points are dense in $\mathbb{C}$.

By Proposition 3, the set $\overline{P(f)}$ is hyperbolic. Hence, for every $z \in \overline{P(f)}$, the sequence $n_{k}=k$ satisfies the claim of Proposition 4. Moreover, $\varepsilon>0$ 
is fixed for such points. If the orbit of some point $z$ eventually enters $\overline{P(f)}$ and $z$ is not pre-critical, then Proposition 4 holds for such a point as well.

Taking now an arbitrary $z \in \mathbb{C}$ whose orbit does not converge to $\infty$ or ever enter $\overline{P(f)}$, we take a compact set $K$ which is visited infinitely often by the orbit and look for an accumulation point $w$ of the orbit, choosing a subsequence so that $f^{n_{k}}(z) \rightarrow w$. If $w \notin \overline{P(f)}$, then for all $k$ large enough a ball of fixed radius centered at $f^{n_{k}}(z)$ is disjoint from $P(f)$, and so can be univalently pulled back to $z$ with bounded distortion.

It remains to consider the case when $w \in \overline{P(f)}$. Let $\gamma_{k}$ be a point of $\overline{P(f)}$ which minimizes the distance to $f^{n_{k}}(z)$. From the hyperbolicity of $\overline{P(f)}$, we can find $m_{k}$ such that a neighborhood of $\gamma_{k}$ is mapped univalently onto $B\left(f^{m_{k}}\left(\gamma_{k}\right), \varepsilon\right)$ and

$$
\frac{\alpha \varepsilon}{\left|f^{n_{k}}(z)-\gamma_{k}\right|}<\left|\left(f^{m_{k}}\right)^{\prime}\left(\gamma_{k}\right)\right|<\frac{16 \varepsilon}{\left|f^{n_{k}}(z)-\gamma_{k}\right|},
$$

where $\alpha, \varepsilon>0$ do not depend on $z$ or $k$. This will guarantee that the preimage of $B\left(f^{m_{k}}\left(\gamma_{k}\right), \varepsilon / 2\right)$ will contain $B\left(\gamma_{k}, 2\left|\gamma_{k}-f^{n_{k}}(z)\right|\right)$. By the lower estimate on the derivative $\left(f^{m_{k}}\right)^{\prime}\left(\gamma_{k}\right)$ and bounded distortion, $f^{m_{k}}$ will map $B\left(f^{n_{k}}(z),\left|f^{n_{k}}(z)-\gamma_{k}\right|\right)$ univalently onto a ball of radius comparable with $\varepsilon$. On the other hand, $B\left(f^{n_{k}}(z),\left|f^{n_{k}}(z)-\gamma_{k}\right|\right)$ is disjoint from $P(f)$, and hence can be pulled back by $f^{n_{k}}$. So, in this case, the sequence $n_{k}+m_{k}$ has the desired property.

This concludes the proof of Proposition 4.

3. Invariant line-fields. Recall that if $M$ is a Riemann surface, then a differential given locally by $\exp (i \theta(z)) d \bar{z} / d z$, where $\theta: M \rightarrow \mathbb{R}$ is measurable, is called a line-field. Since we are working in the measurable category, two line-fields will always be considered equal if they are equal Lebesgue almost everywhere. A non-constant holomorphic function $f$ from $N$ into $M$ has a naturally defined pull-back action of line-fields:

$$
f^{*}\left(\alpha(w) \frac{d \bar{w}}{d w}\right)=\alpha(f(z)) \frac{\bar{f}^{\prime}(z)}{f^{\prime}(z)} \frac{d \bar{z}}{d z} .
$$

Univalently omitted values. To state the main result about non-existence of invariant line-fields, we first need a technical condition.

DeFinition 3. Let $f$ be a meromorphic function on the complex plane. Then $z \in \overline{\mathbb{C}}$ will be called a univalently omitted value for $f$ if every preimage of $z$ is either a critical point of $f$ or belongs to $P(f)$. We will say that $z$ is inherently univalently omitted if $z$ is univalently omitted together with all its forward images.

Obviously, omitted values are univalently omitted as well. 
THEOREM 2. Suppose that $f$ is a meromorphic function whose Julia set is the whole plane, $f$ satisfies the Misiurewicz condition and $\infty \notin P(f)$. Suppose that the set of points whose forward orbits converge to $\infty$ has measure 0. In addition, assume that $f$ has no more than 2 inherently univalently omitted values in the sphere, and if one such value is $\infty$, then the other one is not a pole. Then there is no line-field on the plane invariant under $f^{*}$.

Following the pattern set by [7], our proof will be given in two steps. First, we will show that a potential invariant line-field is analytic on some open set, and then we will prove that a holomorphic line-field cannot exist. More precisely:

Definition 4. A line-field $\nu$ is holomorphic at $z_{0}$ if there is a holomorphic non-constant function $h$ from a neighborhood of $z_{0}$ into $\mathbb{C}$ such that $\nu=h^{*}(d \bar{w} / d w)$ a.e. on this neighborhood. If $h^{\prime}\left(z_{0}\right) \neq 0$, then $\nu$ is univalent.

Proposition 5. Suppose that $f$ is a meromorphic function whose Julia set is the whole plane and satisfies the Misiurewicz condition, $\infty \notin P(f)$ and $\nu$ is a line-field on the plane invariant under $f^{*}$. If the orbit of almost every point does not converge to $\infty$, then there is an open non-empty subset $U \subset \mathbb{C}$ such that $\nu$ is univalent on $U$.

Notice that the hypothesis of Proposition 5 does not contain any restrictions on univalently omitted values. That some additional assumptions in Theorem 2 are necessary is shown by rational examples of Lattés (see $[5$, p. 30]).

The proof of Proposition 5 is standard. Take a point $z$ which is a Lebesgue point of $\nu$ and which also has a sequence $n_{k}$ of passages to the large scale as specified in the claim of Proposition 4. Assume that $f^{n_{k}}(z) \rightarrow w$ and consider $U:=B(w, \varepsilon / 2)$, where $\varepsilon$ comes from Proposition 4 . Define univalent mappings $g_{k}(u)=A_{k}\left(f^{-n_{k}}(u)-z\right)+z$, where $A_{k}$ are positive numbers chosen so that $\left|g_{k}^{\prime}(w)\right|=1$ for all $k$. Then $g_{k}$ converge uniformly on $U$ to a univalent function $G$ with $G(w)=z$ and $\left|G^{\prime}(w)\right|=1$. Consider a constant line-field $\nu_{0}:=\nu(z) d \bar{u} / d u$ and $\nu_{k}(u)=\nu\left(z+A_{k}^{-1}(u-z)\right)$. Since $z$ is a Lebesgue point, $\nu_{k}(u)$ converge to the constant $\nu(z)$ in $L^{1}(G(U))$. For every $k$ we have $\nu=\left(g_{k}^{-1}\right)^{*}\left(\nu_{k} d \bar{u} / d u\right)$. But clearly the coefficient of the line-field on the right-hand side converges to the coefficient of $\left(G^{-1}\right)^{*}\left(\nu_{0}\right)$ in $L^{1}$, and so $\nu$ is univalent as claimed.

3.1. Restrictions on analytic line-fields. In this section we will prove Theorem 2. The proof will be based on two lemmas concerning analytic line-fields. If $\nu=h^{*}(d \bar{w} / d w)$ on some open set and $h$ is holomorphic, we will refer to $h$ as a local linearizing coordinate. 
LEMMA 7. Suppose that $g$ is holomorphic in a neighborhood of a repelling fixed point $z_{0}$. Assume also that there is a line-field $\nu$, holomorphic and invariant in a punctured neighborhood of $z_{0}$. Then there is a function $h$ meromorphic in a neighborhood of $z_{0}$ such that

on that neighborhood.

$$
\nu(z)=(\sqrt{h(z)}) * \frac{d \bar{z}}{d z}
$$

Proof. First, without loss of generality we can assume that $z_{0}=0$ and $g(z)=\lambda z$, where $\lambda=g^{\prime}\left(z_{0}\right)$. Indeed, consider the Koenig coordinate $\phi$ such that $\lambda \phi(z)=\phi(g(z))$. Then once the mapping $h$ postulated in the lemma can be found for the linear map, we can consider $h \circ \phi$ for the original $g$.

If $h_{1}$ and $h_{2}$ are two local linearizing coordinates of $\nu$ with overlapping domains, then $h_{1}^{\prime} / h_{2}^{\prime}$ must be real, hence constant on every connected component of the intersection. It follows that $h_{1}^{\prime \prime} / h_{1}^{\prime}=\left(\log h_{1}^{\prime}\right)^{\prime}$ and $h_{2}^{\prime \prime} / h_{2}^{\prime}$ are equal on the intersection of their domains. This allows us to define a function $N$ to be $h^{\prime \prime} / h^{\prime}$ whenever $h$ is a local linearizing coordinate. Then $N$ is meromorphic in $W$, with poles wherever $h^{\prime}$ vanishes. If we can define a function $H$ on $W$ and $H^{\prime \prime} / H^{\prime}=N$ on $W$, then $(\alpha H)^{*}(d \bar{z} / d z)=\nu$ for a suitably chosen constant $\alpha$.

Invariance of $\nu$ under the action of $g$ means that $N(g(z))\left(g^{\prime}(z)\right)=$ $g^{\prime \prime}(z) / g(z)+N(z)$. Since $g$ is linear, this becomes $N(\lambda z) \lambda=N(z)$. If we consider the function $M(z):=z N(z)$, we get $M(\lambda z)=M(z)$. It follows directly that $M$ is holomorphic in an (unpunctured) neighborhood of 0 , and then that it is constant. So $N(z)=C / z$. If we integrate $N$ along a closed curve surrounding 0 , we should get the difference of the logarithms of the derivatives of two linearizing coordinates of $\nu$. This difference is $\pi$ times an integer (not necessarily an even one, because the derivatives could have opposite signs). Hence, $2 C$ is an integer. Then if we set $H(z)=z^{C+1}$, we get $N=h^{\prime \prime} / h^{\prime}$ and hence by the remark of the preceding paragraph, $h=\alpha H$ will have the postulated property.

\subsection{Propagation of an analytic line-field}

LEMMA 8. Suppose that $f$ is a meromorphic function on the complex plane, $f$ satisfies the Misiurewicz condition, $\infty \notin P(f)$, and the Julia set of $f$ is the whole plane. Assume that $f$ fixes a line-field $\mu$ on the plane, which is holomorphic in some open set $U$. Then $\mu$ is holomorphic at every point of the Riemann sphere except for the inherently univalently omitted values of $f$.

Proof. It clearly suffices to prove that $\mu$ is holomorphic at every value which is not univalently omitted. Then, if $a$ has a forward image which is not univalently omitted, the field can be pulled back by $f$ from a neighborhood of that point. 
So suppose that $a$ is not univalently omitted. Then it has a preimage $b$, other than itself, which is not a critical point of $f$ and not in $P(f)$. The only thing needed to be proved in addition to what follows from Definition 3 is that $b \neq a$. If $a \in P(f)$, that follows automatically. If $a \notin P(f)$, then none of its preimages is a critical point or an element of $P(f)$, so the only problem occurs if $a$ is omitted or $a$ is its only preimage. In both cases, $a$ is asymptotic by Iversen's theorem, and so $a \in P(f)$, resulting in a contradiction.

Since $b \notin P(f)$, its preimages are neither critical points nor in $P(f)$. There must be a preimage other than $a, b$. Indeed, it could only be $a$, since $f(b)=a$, but again if $b$ has at most one preimage it would be asymptotic. By the same method, we choose $d$ which is different from $a, b, c$ and $f(d)=c$. Since we have three distinct points $b, c, d$, at least one of them is attained by some iterate of $f$ on $U$. Since all these points are outside $\operatorname{Sing}(f)$, this iterate has a local inverse allowing one pull back $\mu$ from $U$ and thus establish its univalence at this point. But then we can map forward to $a$ by at most three iterates of $f$ which are also locally univalent. Hence, $\mu$ is univalent at $a$ as needed, and in the light of the opening remark, Lemma 8 follows.

Proof of Theorem 2. By Lemma 8, $\nu$ is holomorphic except at the inherently univalently omitted values of $f$. Moreover, since a holomorphic field can always be pulled back by $f$, the only possible points of non-holomorphy for $f$ are even more restricted, to those inherently univalently omitted values which are either at $\infty$ or mapped into another inherently univalently omitted value. So, if $E$ denotes the set of points in $\mathbb{C}$ where $\nu$ is not holomorphic, then $f(E) \subset E$. This in particular follows from the fact that $\infty \notin f(E)$ even if $\infty$ is univalently omitted, by the hypothesis of Theorem 2 for the case when $\infty$ is univalently omitted. So, $E$ consists of periodic orbits of $f$, which must be repelling by Proposition 3. Using Lemma 7, we can determine the kind of singularities of the field at those points. Consider the Riemann surface $\widetilde{M}$ incorporating all local linearizing coordinates of $\nu$. This $\widetilde{M}$ is the covering of either $\mathbb{C}$ with one point removed, or the sphere $\overline{\mathbb{C}}$ with two points removed. Those singularities are in fact branching points of degree 2 , and if $M$ denotes $\widetilde{M}$ completed by adding those branching points, then there is a meromorphic function $H: M \rightarrow \overline{\mathbb{C}}$ such that $\pi^{*}(\mu)=H^{*}(d \bar{z} / d z)$. Here $\pi$ is the natural projection from a Riemann surface of a function. In fact, $(M, \pi)$ is then isomorphic to the branched covering of either the sphere or the plane by the quadratic root, in particular $M$ is either the sphere or the plane.

We claim that $f$ lifts to a function $F: M \backslash \pi^{-1}(\infty) \rightarrow M$. We will discuss in detail only the more complicated case of two branching points. One needs to check the standard lifting condition for the map $f \circ \pi$ from $\widetilde{M} \backslash \pi^{-1}(\infty)$ into $\overline{\mathbb{C}} \backslash E$ to the unbranched covering $(\widetilde{M}, \pi)$. The image of the fundamental 
group of $\widetilde{M} \backslash \pi^{-1}(\infty)$ is the set of loops in $\mathbb{C}$ whose indices with respect to the two points in $E$ differ by an even number. By the argument principle, their images under $f$ will have the same property, but that means they are in $\pi_{*}\left(\pi_{1}(\widetilde{M})\right)$.

The function $F$ preserves the holomorphic line-field $H^{*}(d \bar{z} / d z)$. This means that the map $H \circ F \circ H^{-1}$ is locally affine. Since $F$ and $H$ are, however, globally defined, that means $H \circ F=A \circ H$ for some affine map $A$. To finish the proof, note that because the covering by $M$ is only two-sheeted, each point periodic under $f$ contains in its fiber a point which is periodic for $F$, perhaps with the period doubled. Considering a repelling periodic point $q_{1}$ of period $p_{1}$, we see that $A^{p_{1}}\left(H\left(q_{1}\right)\right)=H\left(q_{1}\right)$. But the same will apply to any other periodic point $q_{2}$ with period $p_{2}$, which would lead to the conclusion that $A^{p_{1} p_{2}}$ is the identity, which contradicts the fact these periodic points can be chosen from the Julia set and hence repelling.

This contradiction concludes the proof of Proposition 5 and hence of Theorem 2.

\section{References}

[1] I. N. Baker, J. Kotus and Y. Lü, Iterates of meromorphic functions I, Ergodic Theory Dynam. Systems 11 (1991), 241-248.

[2] - - - - - Iterates of meromorphic functions IV, Results Math. 22 (1992), 651-656.

[3] W. Bergweiler, Iteration of meromorphic functions, Bull. Amer. Math. Soc. 29 (1993), 151-188.

[4] W. Bergweiler and A. Eremenko, On the singularities of the inverse to a meromorphic function of finite order, Rev. Mat. Iberoamericana 11 (1995), 355-373.

[5] L. Carleson and T. Gamelin, Complex Dynamics, Springer, New York, 1993.

[6] A. A. Gol'dberg and I. V. Ostrovskiǔ, Distribution of Values of Meromorphic Functions, Nauka, Moscow 1970 (in Russian).

[7] C. McMullen, Renormalization and 3-Manifolds which Fiber over the Circle, Ann. of Math. Stud. 142, Princeton Univ. Press, 1998.

[8] P. J. Rippon and G. M. Stallard, Iteration of a class of hyperbolic meromorphic functions, Proc. Amer. Math. Soc. 127 (1999), 3251-3258.

Département de Mathématiques

Université de Paris-Sud

91405 Orsay, France

E-mail: graczyk@math.u-psud.fr

Department of Mathematics

Penn State University

University Park, PA 16802, U.S.A.

E-mail: swiatek@math.psu.edu
Department of Mathematics Warsaw University of Technology 00-661 Warszawa, Poland E-mail: janinak@impan.gov.pl

Received 2 July 2004; in revised form 17 September 2004 